\title{
Rescue of multiple class II CFTR mutations by elexacaftor+tezacaftor+ ivacaftor mediated in part by the dual activities of elexacaftor as both corrector and potentiator
}

\author{
Onofrio Laselva (10,2, Claire Bartlett ${ }^{3}$, Tarini N.A. Gunawardena ${ }^{1,3}$, \\ Hong Ouyang ${ }^{3}$, Paul D.W. Eckford ${ }^{1}$, Theo J. Moraes ${ }^{3,4}$, Christine E. Bear ${ }^{1,2,5}$ and \\ Tanja Gonska ${ }^{3,4}$
}

Affiliations: ${ }^{1}$ Programme in Molecular Medicine, The Hospital for Sick Children, Toronto, ON, Canada. ${ }^{2}$ Dept of Physiology, University of Toronto, Toronto, ON, Canada. ${ }^{3}$ Programme in Translational Medicine, The Hospital for Sick Children, Toronto, ON, Canada. ${ }^{4}$ Dept of Paediatrics, University of Toronto, Toronto, ON, Canada. ${ }^{5}$ Dept of Biochemistry, University of Toronto, Toronto, ON, Canada.

Correspondence: Tanja Gonska, Programme in Translational Medicine, The Hospital for Sick Children, Room 8415, 555 University Avenue, Toronto, ON M5G 8X4, Canada. E-mail: tanja.gonskaldsickkids.ca

@ERSpublications

Using pre-clinical studies of F508del in nasal cultures as a benchmark, this study identified other CF mutations for which Trikafta may be clinically effective https://bit.ly/3mbMOqL

Cite this article as: Laselva O, Bartlett C, Gunawardena TNA, et al. Rescue of multiple class II CFTR mutations by elexacaftor+tezacaftor+ivacaftor mediated in part by the dual activities of elexacaftor as both corrector and potentiator. Eur Respir J 2021; 57: 2002774 [https://doi.org/10.1183/13993003.02774-2020].

ABSTRACT Positive results in pre-clinical studies of the triple combination of elexacaftor, tezacaftor and ivacaftor, performed in airway epithelial cell cultures obtained from patients harbouring the class II cystic fibrosis transmembrane conductance regulator (CFTR) mutation F508del-CFTR, translated to impressive clinical outcomes for subjects carrying this mutation in clinical trials and approval of Trikafta.

Encouraged by this correlation, we were prompted to evaluate the effect of the elexacaftor, tezacaftor and ivacaftor triple combination on primary nasal epithelial cultures obtained from individuals with rare class II CF-causing mutations (G85E, M1101K and N1303K) for which Trikafta is not approved.

Cultures from individuals homozygous for M1101K responded better than cultures harbouring G85E and $\mathrm{N} 1303 \mathrm{~K}$ after treatment with the triple combination with respect to improvement in regulated channel function and protein processing. A similar genotype-specific effect of the triple combination was observed when the different mutations were expressed in HEK293 cells, supporting the hypothesis that these modulators may act directly on the mutant proteins. Detailed studies in nasal cultures and HEK293 cells showed that the corrector, elexacaftor, exhibited dual activity as both corrector and potentiator, and suggested that the potentiator activity contributes to its pharmacological activity.

These pre-clinical studies using nasal epithelial cultures identified mutation genotypes for which elexacaftor, tezacaftor and ivacaftor may produce clinical responses that are comparable to, or inferior to, those observed for F508del-CFTR.

This article has supplementary material available from erj.ersjournals.com

Received: 11 April 2020 | Accepted: 20 Nov 2020

Copyright OERS 2021. This version is distributed under the terms of the Creative Commons Attribution NonCommercial Licence 4.0. 


\section{Introduction}

Cystic fibrosis (CF) is caused by mutations in the CF transmembrane conductance regulator (CFTR) gene, which encodes for an ion channel mediating chloride and bicarbonate transport across epithelial cells. $\mathrm{CF}$ patients suffer from multiorgan disease; however, early mortality is mainly caused by progressive, destructive lung disease [1-3]. Following the clinical success of the first CFTR modulators, ivacaftor (VX-770) and lumacaftor (VX-809) [4-6], the US Food and Drug Administration (FDA) has recently approved the next-generation CFTR modulator Trikafta. This highly effective CFTR modulator consists of two corrector molecules, elexacaftor (VX-445) and tezacaftor (VX-661), which synergistically help to process misfolded CFTR protein to the cell membrane, and a potentiator, ivacaftor, which increases channel opening $[7,8]$. Phase 3 clinical trials with Trikafta have shown dramatic improvement in lung function, reduction in pulmonary exacerbations and improvement in the quality of life of patients carrying at least one c.1521_1523delCTT (F508del)-CFTR allele [7, 9].

F508del-CFTR is the prototypical class II CFTR mutation resulting in defective CFTR protein trafficking due to protein misfolding, reduced stability of the protein at the cell surface and dysfunctional channel gating [10]. There are rare class II mutations such as c.3302T>A (M1101K), c.254G $>$ A (G85E), c.1705T $>$ G (Y569D) and c.3909C > G (N1303K), which are found in $0.2 \%, 0.7 \%, 0.03 \%$ and $2.4 \%$ of CFTR2 registered $\mathrm{CF}$ patients, respectively (https://cftr2.org). It has been previously reported that some of these rare mutations (i.e. M1101K, G85E and N1303K) show a defect in CFTR processing, similar to F508del-CFTR $[11,12] . \mathrm{M} 1101 \mathrm{~K}$ and N1303K mutations demonstrated only a modest rescue after lumacaftor correction and ivacaftor potentiation when expressed in heterologous expression systems [13-15].

In the current work, we aimed to evaluate the effect of Trikafta on rare class II CFTR mutations using nasal epithelial cell cultures generated from CF patients homozygous for M1101K, G85E and N1303K.

\section{Material and methods \\ Patients}

Nasal epithelial cell cultures were obtained from CF patients enrolled in the Canadian Program for Individualised CF Therapy (CFIT) (https://lab.research.sickkids.ca/cfit) [16]. This study was approved by the Research Ethics Board of The Hospital for Sick Children and St Michael's Hospital (REB1000044783) (Toronto, ON, Canada). All study participants or their guardians signed an informed consent.

\section{Cell culture}

Human embryonic kidney 293 GripTite cells (HEK239) were maintained in DMEM (Wisent, St-Bruno, QC, Canada) supplemented with nonessential amino acids (Life Technologies, Waltham, MA, USA) and $10 \%$ fetal bovine serum (Wisent) at $37^{\circ} \mathrm{C}$ and $5 \% \mathrm{CO}_{2}$ as previously described [17]. All CFTR variants used in this study were transiently expressed in HEK293 cells using PolyFect Transfection Reagent (Qiagen, Hilden, Germany), according to the manufacturer's protocol as previously described [18].

16HBE14o- cells, CRISPR/Cas9 edited to express N1303K-CFTR, were obtained from the Cystic Fibrosis Foundation (CFF 16HBEge N1303K-CFTR) [19]. Nasal epithelial cell cultures were generated following nasal brushing of the individual patients as previously described $[20,21]$.

Nasal epithelial cells were expanded and frozen at passage 1 . These passage 1 cells were thawed and expanded in passage 2 using PneumaCult-Ex Plus (STEMCELL Technologies, Vancouver, BC, Canada) and then seeded on collagen-coated Transwell inserts as passage $3(6.5 \mathrm{~mm}$ diameter, $0.4 \mu \mathrm{m}$ pore size; Corning, Tewksbury, MA, USA). Once confluent, the cells were cultured for 14 days at an air-liquid interface (ALI) with basal differentiation media (PneumaCult-ALI; STEMCELL Technologies) [16, 22, 23] before functional and protein studies were performed.

\section{CFTR channel function in HEK293 and CFF-16HBE140- N1303K-CFTR cells}

HEK293 cells were seeded in 96-well plates (Costar; Corning). After $24 \mathrm{~h}$ the cells were transfected with either M1101K-, G85E- or N1303K-CFTR constructs and $18 \mathrm{~h}$ post-transfection were treated with the following CFTR modulators: $0.1 \%$ dimethyl sulfoxide (DMSO), $3 \mu \mathrm{M}$ VX-809, $3 \mu \mathrm{M}$ VX-809 or $3 \mu \mathrm{M}$ $S$-VX $445+3 \mu \mathrm{M} \mathrm{VX}-661$ for $24 \mathrm{~h}$ at $37^{\circ} \mathrm{C}$. $16 \mathrm{HBE} 14 \mathrm{o}-$ cells were grown at $37^{\circ} \mathrm{C}$ for 5 days post-confluence on 96-well plates (Costar) as previously described [24]. The cells were treated with the aforementioned CFTR modulators $24 \mathrm{~h}$ before fluorometric imaging plate reader functional assay (FLIPR; Molecular Devices, San Jose, CA, USA). Cells were then loaded with blue FLIPR membrane potential dye dissolved in chloride-free buffer (136 mM sodium gluconate, $3 \mathrm{mM}$ potassium, gluconate, $10 \mathrm{mM}$ glucose, $20 \mathrm{mM}$ HEPES, pH 7.35, $300 \mathrm{mOsm}$, at a concentration of $0.5 \mathrm{mg} \cdot \mathrm{mL}^{-1}$ ) for $30 \mathrm{~min}$ at $37^{\circ} \mathrm{C}$. CFTR function was determined using FLIPR Tetra at $37^{\circ} \mathrm{C}$. After establishing a baseline fluorescence read (excitation $530 \mathrm{~nm} /$ emission $560 \mathrm{~nm}$ ) for $5 \mathrm{~min}$, CFTR was stimulated using forskolin (FSK) (10 $\mu \mathrm{M}$; Sigma) and the potentiators VX-770 $(1 \mu \mathrm{M})$ or $S$-VX-445 $(1 \mu \mathrm{M})$. CFTR-mediated depolarisation of the plasma membrane 
was detected as an increase in fluorescence following which the CFTR inhibitor CFTRinh-172 $(10 \mu \mathrm{M}$; EMD Millipore, Burlington, MA, USA) was added to inactivate CFTR. The peak changes in fluorescence to CFTR agonists were normalised relative to the baseline fluorescence $\left(\Delta F / F_{0}\right)[24,25]$.

\section{Ussing chamber studies of primary nasal epithelial cells}

Day 14 ALI nasal epithelial cells in 6.5-mm transwells were mounted in circulating Ussing chambers (Physiological Instruments, San Diego, CA, USA) and measurements to assess CFTR function were performed as previously described [26]. The perfusion bath $(126 \mathrm{mM} \mathrm{NaCl}, 24 \mathrm{mM} \mathrm{NaHCO}, 2.13 \mathrm{mM}$ $\mathrm{K}_{2} \mathrm{HPO}_{4}, 0.38 \mathrm{mM} \mathrm{KH}_{2} \mathrm{PO}_{4}, 1 \mathrm{mM} \mathrm{MgSO} 41 \mathrm{mM} \mathrm{CaCl}_{2}$ and $10 \mathrm{mM}$ glucose) was maintained at a $\mathrm{pH}$ of 7.4, a temperature of $37^{\circ} \mathrm{C}$ and continuously gassed with a $5 \% \mathrm{CO}_{2} / 95 \% \mathrm{O}_{2}$ mixture. Measurements were performed in symmetrical chloride concentrations, in open-circuit mode, and are presented as transepithelial current $\left(I_{\text {eq }} ; \mu \mathrm{A} \cdot \mathrm{cm}^{-2}\right)$. The transepithelial resistance was $250 \pm 122 \Omega \cdot \mathrm{cm}^{-2}$ for healthy control nasal cells and $330 \pm 130 \Omega \cdot \mathrm{cm}^{-2}$ for CF nasal cells. Before the experiments, cells were treated with $0.1 \%$ DMSO, $3 \mu \mathrm{M}$ VX-809+1 $\mu \mathrm{M}$ VX-770, $3 \mu \mathrm{M}$ VX-661+3 $\mu \mathrm{M} R$-VX-445+1 $\mu \mathrm{M}$ VX-770 or $3 \mu \mathrm{M}$ VX-661+3 $\mu \mathrm{M} S$-VX-445+1 $\mu \mathrm{M}$ VX-770 for $48 \mathrm{~h}$. During the experiment $1 \mu \mathrm{M}$ VX-770 was also added acutely. CFTR function was determined in the presence of amiloride $(30 \mu \mathrm{M}$; Spectrum Chemical, Gardena, CA, USA) and following cAMP activation with FSK ( $I_{\text {eq }}$ FSK, 0.1 or $10 \mu \mathrm{M}$ for wild-type (WT) or $10 \mu \mathrm{M}$ for F508del-, M1101K-, G85E- and N1303K-CFTR; Sigma-Aldrich, St Louis, MO, USA). CFTR activity was confirmed as $I_{\mathrm{eq}}$ difference following CFTR inhibition with CFTRinh-172 ( $I_{\mathrm{eq}}$ CFTRinh-172, $10 \mu \mathrm{M})[20,27]$. The maximal response $I_{\text {eq }}$ FSK was calculated as the difference between the steady baseline of the measured $I_{\mathrm{eq}}$ following amiloride inhibition and the lowest measured peak $I_{\mathrm{eq}}$ following addition of ivacaftor/elexacaftor plus FSK.

\section{Immunoblotting}

Day 14 ALI nasal epithelial cells were lysed in modified radioimmunoprecipitation assay buffer $(50 \mathrm{mM}$ Tris-HCl, $150 \mathrm{mM} \mathrm{NaCl}, 1 \mathrm{mM}$ EDTA, pH 7.4, 0.2\% SDS and 0.1\% Triton X-100) containing a protease inhibitor cocktail (Roche, Mannheim, Germany) for $10 \mathrm{~min}$ [28]. Soluble fractions were analysed by SDSPAGE on 6\% Tris-glycine gels (Life Technologies, Carlsbad, CA, USA). After electrophoresis, the proteins were transferred to nitrocellulose membranes (Bio-Rad, Hercules, CA, USA) and incubated in 5\% milk. CFTR bands were detected with human CFTR-specific murine mAb 596 (nucleotide binding domain (NBD) 2, 1024-1211; University of North Carolina, Chapel Hill, NC, USA) using a 1:500 dilution. The blots were developed with enhanced chemiluminescent agent (ECL; Amersham, Little Chalfont, UK) using the Odyssey Fc imaging system (LI-COR Biosciences, Lincoln, NE, USA) in a linear range of exposure (2$45 \mathrm{~min}$ ) [29-31]. Relative levels of CFTR protein were quantified by densitometry of immunoblots using ImageStudioLite (LI-COR Biosciences).

\section{Compound description}

VX-809, VX-661 and VX-770 were from Selleck Chemicals (Houston, TX, USA); R-VX-445 (N-(1,3-dimethylpyrazol-4-yl)sulfonyl-6-(3-(3,3,3-trifluoro-2,2-dimethyl-propoxy)pyrazol-1-yl)-2-((4R)-2,2,4-trimethylpyrrolidin-1-yl)pyridine-3-carboxamide) and S-VX-445 (N-(1,3-dimethylpyrazol-4-yl)sulfonyl-6-(3-(3,3, 3-trifluoro-2,2-dimethyl-propoxy)pyrazol-1-yl)-2-((4S)-2,2,4-trimethylpyrrolidin-1-yl)pyridine-3-carboxamide) were from MedChemExpress (Monmouth Junction, NJ, USA) [24].

\section{Statistical analysis}

Data are presented as mean with standard deviation. The paired two-tailed t-test or one-way ANOVA was used for comparison between groups with a significance level of $\mathrm{p}<0.05$. Data with multiple comparisons were assessed using Tukey's multiple comparison test with $\alpha=0.05$. Prism version 7.0 (GraphPad, San Diego, CA, USA) was used for all statistical tests.

\section{Results}

Triple modulator combination VX-445+VX-661+VX-770 partially rescues the processing and functional defect of F508del in nasal epithelial cell cultures

As the triple combination VX-445+VX-661+VX-770 was approved by the FDA for the CFTR mutant F508del, we first assessed the CFTR response to this drug combination in patient-derived nasal epithelial cells from three F508del/F508del patients as a benchmark experiment. Due to the absence of detailed information on the enantiomer of VX-445 used in the clinically approved triple drug combination, we tested both the $R$ - and $S$-isoforms of VX-445. Treatment with $S$-VX-445+VX-661+VX-770 showed a statistically significant increase in $I_{\text {eq }}$ after acute application of FSK and VX-770 in F508del/F508del nasal epithelial cells and an even larger increase in $I_{\text {eq }}$ CFTRinh-172, a result that is consistent with enhanced constitutive CFTR channel activity following chronic treatment with the potentiator, VX-770. The $R$-enantiomer was less effective in modulating this response (figure 1a-c). Experiments were conducted 
a)

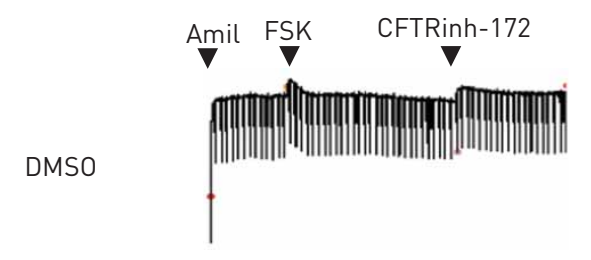

$V X-809$

$+\mathrm{VX}-770$
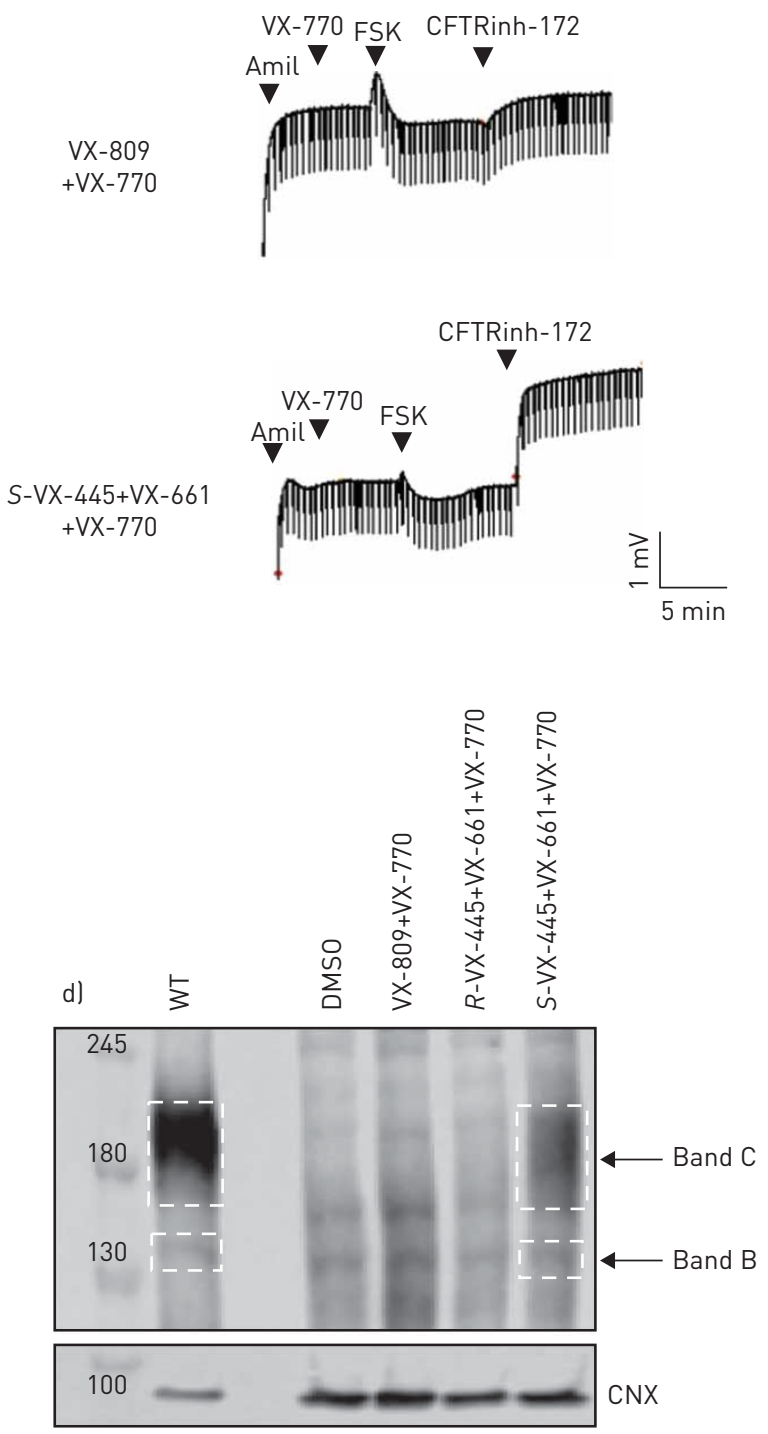
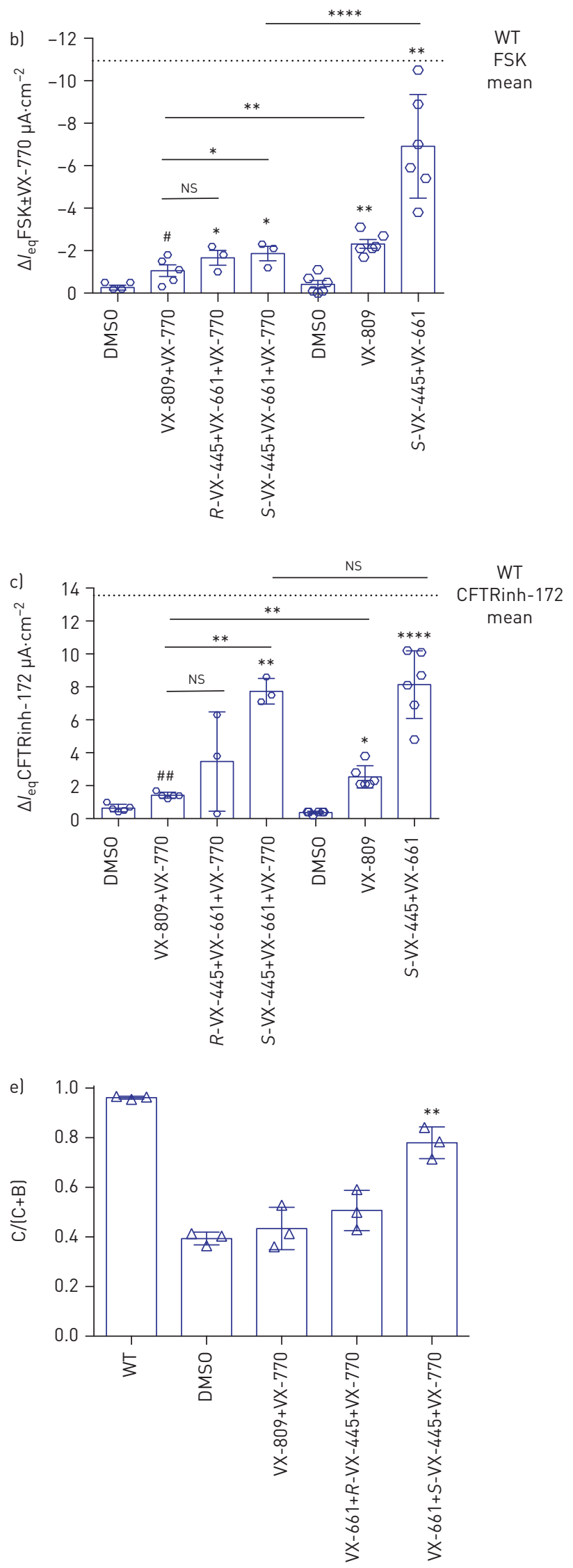
FIGURE 1 F508del cystic fibrosis transmembrane conductance regulator (CFTR) mutation is rescued by the triple combination VX-445+VX-661 $+V X-770$ in nasal epithelial cells from three patients homozygous for F508del. DMSO: dimethyl sulfoxide; amil: amiloride; FSK: forskolin; $I_{\text {eq: }}$ transepithelial current; WT: wild-type; CNX: calnexin. a) Representative tracings show Ussing chamber measurements of CFTR function in nasal epithelial cell cultures from a CF patient bearing F508del/F508del in the absence or presence of the small-molecule corrector. The upper line reflects the transepithelial potential difference measurements and the downward deflection reflects the transepithelial resistance. b) Maximal response $l_{\text {eq }}$ after stimulation with FSK $(10 \mu \mathrm{M}) \pm \mathrm{VX}-770(1 \mu \mathrm{M})$ of one or two technical replicate experiments of nasal cultures generated from three patients. Different pre-treatments were performed (48 h at $\left.37^{\circ} \mathrm{C}\right)$ : DMSO $(0.1 \%), \mathrm{VX}-809(3 \mu \mathrm{M})+\mathrm{VX}-770(1 \mu \mathrm{M}), R-V X-445(3 \mu \mathrm{M})+\mathrm{VX}-661$ $(3 \mu \mathrm{M})+V X-770(1 \mu \mathrm{M}), S-V X-445(3 \mu \mathrm{M})+V X-661(3 \mu \mathrm{M})+V X-770(1 \mu \mathrm{M}), V X-809(3 \mu \mathrm{M})$ or $R-V X-445(3 \mu \mathrm{M})+V X-661(3 \mu \mathrm{M})$. The data points show single Ussing chamber experiments. Comparative analysis showed statistically significant differences between nasal epithelial cells treated with VX-809+VX-770 and VX-445+VX-661 as well as those treated with acute and chronic VX-770. c) / eq by the CFTR inhibitor CFTRinh-172 (10 $\mu$ M) from the same experiments done in b). d) Immunoblots of steady-state expression of WT or F508del following treatments with CFTR modulators. Band C: mature, complex-glycosylated CFTR; band B: immature, core-glycosylated CFTR. Dash-lined boxes provide an example how the area for quantification of the protein abundance was chosen. Markers in $\mathrm{kDa}$. e) Ratio band $\mathrm{C} /($ band $\mathrm{C}+$ band $\mathrm{B})$ of measured nasal epithelial cells from three patients. Comparative analysis showed statistically significant differences between nasal epithelial cells treated with DMSO and VX-445+ VX-661 \pm VX-770. Data are presented as mean \pm SD. One-way ANOVA followed by Turkey's post hoc test was used for statistical analysis (*: $p<0.05$; $\left.{ }^{* *}: \mathrm{p}<0.01 ; * * * *: \mathrm{p}<0.0001\right)$. The paired t-test showed statistically differences between nasal epithelial cells treated with DMSO and VX-809+VX-770 $\left.{ }^{\#}: p<0.05 ;{ }^{\# \#}: p<0.01\right)$. NS: nonsignificant.

with chronic and acute application of VX-770 demonstrating that if VX-770 is added chronically, the potentiated FSK response is smaller than the response if VX-770 is added acutely, together with FSK (figure $1 \mathrm{~b}$ and $\mathrm{c}$ ). This is consistent with reports of the inhibitory effect of chronic VX-770 on the functional expression of F508del-CFTR (figure 1a-c) [32, 33]. As chronic VX-770 treatment is thought to better reflect the clinical pharmacokinetics of these combination drugs, we continued testing the effect of CFTR modulators with chronic application of VX-770. In order to show that the combination of VX-445 and VX-661, and not just VX-661, caused the larger responses when compared with VX-809 treatment, we performed comparative experiments in F508del/F508del nasal epithelial cells between VX-809 and VX-661, and demonstrated no difference between the response (supplementary figure S1). Since VX-809 is an integral part of the clinically approved drug Orkambi, we continued use of VX-809 for the rest of the experiments.

Western blot analysis of the treated F508del/F508del nasal epithelial cell cultures showed a significant increase in the mature form (band C) of the F508del-CFTR protein following treatment with S-VX-445+ VX-661+VX-770 compared with DMSO treatment and was $\sim 70 \%$ of WT-CFTR protein processing (band $\mathrm{C} /($ band $\mathrm{C}+$ band $\mathrm{B})$ ). The $R$-isoform of VX-445 had no effect on CFTR protein expression. These findings support correction of the processing defect of F508del-CFTR in patient-derived nasal epithelial cells by $S$-VX-445+VX-661+VX-770 resulting in improved CFTR function (figure $1 \mathrm{~d}$ and e). In order to compare drug responses between different CFTR mutations in a clinically relevant manner, we used the F508del/F508del results as a benchmark to study the effect of the triple combination on other class II mutations. Since the initiation of these experiments it has been confirmed that the $S$-enantiomer is the clinical compound.

\section{Variable levels of VX-445+VX-661+VX-770 rescue are observed for other class II mutations}

We next studied the effect of VX-445+VX-661+VX-770 on CFTR function in nasal epithelial cell cultures from patients homozygous for M1101K, G85E or N1303K. Treatment of nasal epithelial cultures from three individuals homozygous for M1101K with S-VX-445+VX-661+VX-770 led to a significant increase in CFTR function, measured as the acute current response to FSK plus potentiator or as the increase in $I_{\mathrm{eq}}$ CFTRinh-172 (figure 2a, g and $\mathrm{h}$ ). The CFTR response to triple combination exceeded the response observed in nasal epithelial cells from three F508del/F508del patients. This functional rescue is supported by an increase in mature CFTR protein. Western blot analysis showed a significant increase in M1101K-CFTR protein processing (band $\mathrm{C} /($ band $\mathrm{C}+$ band B)) following treatment with S-VX-445+ VX-661+VX-770 relative to DMSO (figure $2 b$ and $j$ ). Triple combination with the $R$-enantiomer also increased M1101K-CFTR function, but to a lesser extent (figure $2 \mathrm{~g}, \mathrm{~h}$ and $\mathrm{j}$ ).

Treatment of nasal epithelial cells from three subjects homozygous for G85E with S-VX-445+VX-661+ VX-770 showed a small but significant increase in CFTR function, when measured as the $I_{\text {eq }}$ CFTRinh-172-sensitive current and when compared with DMSO-treated nasal cells. The S-VX-445+ VX-661+VX-770-mediated increase in $I_{\text {eq }}$ CFTRinh-172 reached about half of the $I_{\text {eq }}$ CFTRinh-172 increase measured in F508del/F508del nasal epithelial cells (figure 2c, g, h and i). This increase in CFTR function was paralleled by a small but significant increase in G85E-CFTR protein maturation relative to DMSO pre-treatment (figure $2 \mathrm{~d}$ and $\mathrm{j}$ ). In this mutation, triple combination with the $R$-enantiomer had no significant effect (figure $2 \mathrm{~g}, \mathrm{~h}$ and $\mathrm{j}$ ).

Nasal cells from two patients homozygous for N1303K showed a significant increase in CFTR function measured as $I_{\text {eq }}$ FSK and $I_{\text {eq }}$ CFTRinh-172 following $48 \mathrm{~h}$ of treatment with $S$-VX-445+VX-661+VX-770 
a)

DMSO

VX-809
$+V X-770$

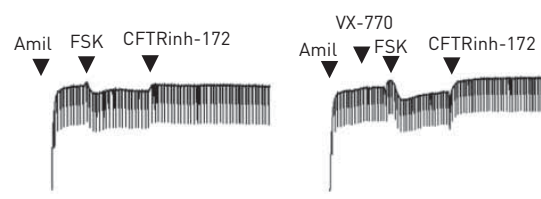

c)

DMSO

$V X-809$
$+V X-770$

e)

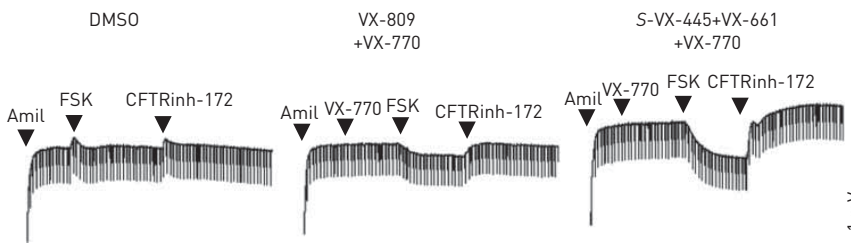

Amil FSK CFTRinh-172

(m)

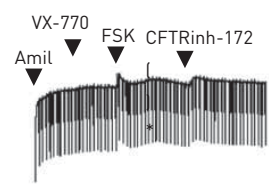

$\stackrel{\gtrless}{-} L_{5 \mathrm{~min}}$

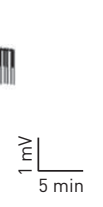

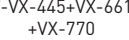

$S-V X-445+V X-66$

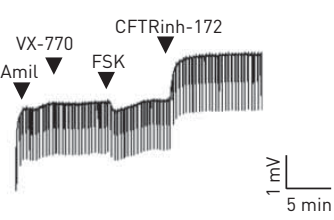

b)

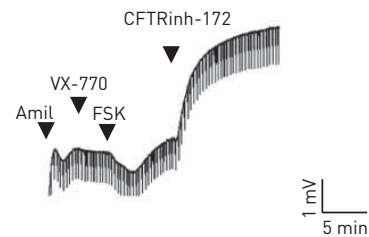

d)
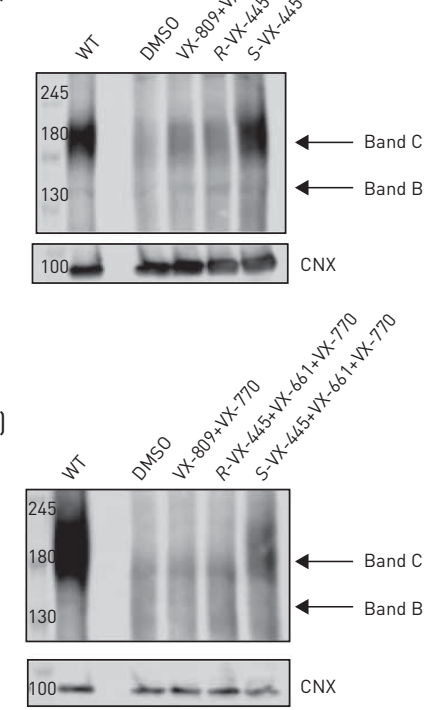

f)
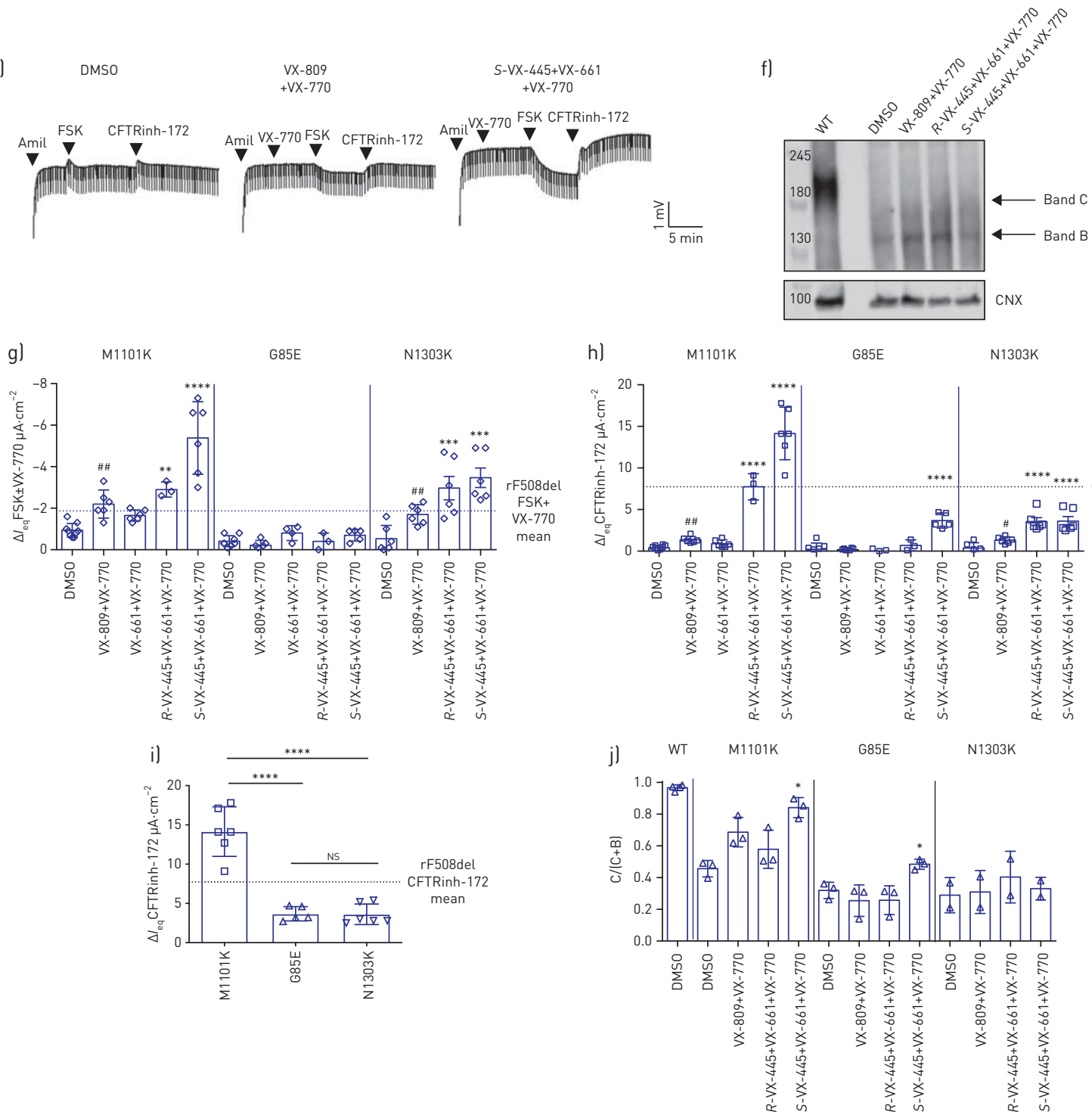
FIGURE 2 Nasal epithelial cultures derived from patients homozygous for other class II cystic fibrosis transmembrane conductance regulator (CFTR) mutations exhibit low CFTR function and variable levels of rescue by VX-445+VX-661+VX-770. DMSO: dimethyl sulfoxide; amil: amiloride; FSK: forskolin; CNX: calnexin; leq: transepithelial current; WT: wild-type. a-f) Representative tracings show Ussing chamber measurements of CFTR function in nasal epithelial cell cultures from CF patients bearing a) M1101K/M1101K, c) G85E/G85E and e) N1303K/N1303K in the absence or presence of the small-molecule corrector. Immunoblots of steady-state expression of WT and b) M1101K, d) G85E and f) N1303K following treatments with CFTR modulators. Markers in kDa. Band C: mature, complex-glycosylated CFTR; band B: immature, core-glycosylated CFTR. g) Maximal response $l_{\text {eq }}$ after stimulation by FSK $(10 \mu \mathrm{M}) \pm \mathrm{VX}-770(1 \mu \mathrm{M})$ for nasal epithelial cell cultures from one to three technical replicates of three patients bearing M1101K or G85E and two donors bearing N1303K after pre-treatment ( $48 \mathrm{~h}$ at $\left.37^{\circ} \mathrm{C}\right)$ with $\mathrm{DMSO}(0.1 \%), \mathrm{VX}-809(3 \mu \mathrm{M})+$ VX-770 $(1 \mu \mathrm{M}), R$-VX-445 $(3 \mu \mathrm{M})+V X-661(3 \mu \mathrm{M})+V X-770(1 \mu \mathrm{M})$ or $S-V X-445(3 \mu \mathrm{M})+V X-661(3 \mu \mathrm{M})+V X-770(1 \mu \mathrm{M})$. h) leg by the CFTR inhibitor CFTRinh-172 $(10 \mu \mathrm{M})$ for nasal epithelial cell cultures from one to three technical replicates of three patients bearing M1101K or G85E and two donors bearing N1303K. i) Comparison of $l_{\text {eq }}$ CFTRinh-172 across the class II mutations M1101K, G85E and N1303K for nasal epithelial cell cultures from one to three technical replicates of three patients bearing M1101K or G85E and two donors bearing N1303K. Dashed line in $\mathrm{g}$-i) represents the mean results of rescued (r) F508del/F508del nasal cells treated with VX-445+VX-661+VX-770. j) Ratio band C/(band C+band B) from one technical replicate of three patients bearing M1101K or G85E and two patients bearing N1303K. Data are presented as mean \pm SD. Analysis was performed using one-way ANOVA followed by Turkey's post hoc test $\left(*: p<0.05 ; *^{* *}: p<0.01 ;{ }^{* * *}: p<0.001 ;{ }^{* * * *}: p<0.000\right)$. The paired t-test showed statistically differences between nasal epithelial cells treated with DMSO and VX-809+VX-770 ("\#: $p<0.05$; \#\#: $p<0.01$ ). NS: nonsignificant.

and also acute addition of VX-770 (figure 2e, $\mathrm{g}$ and $\mathrm{h}$ ). However, interestingly, there was negligible improvement in N1303K-CFTR protein processing caused by $S$-VX-445+VX-661+VX-770 on N1303K-CFTR protein (figure $2 \mathrm{f}$ and $\mathrm{j}$ ). This suggests that the increase in N1303K-CFTR function did not occur via improved protein processing, rather by potentiation of the function of membrane-residual N1303K proteins. Also, it was interesting to note that the triple combination treatment with both the $S$ and the $R$-enantiomer had a similar effect.

\section{VX-445 functions as a corrector and a potentiator drug}

One way to explain the discrepancy between the VX-445+VX-661+VX-770-induced increase in N1303K-CFTR function and protein expression is that the combination drugs, perhaps VX-445, exhibit potentiator activity. To test the hypothesis that, in addition to its corrector activity, VX-445 functions as a CFTR potentiator, we first examined the effect of acute addition of $S$-VX-445 on channel function of WT-CFTR using nasal and bronchial epithelial cells derived from non-CF controls and non-CF lung explants, respectively. Interestingly, the acute addition of $S$-VX-445 increased $I_{\mathrm{eq}}$ FSK in both WT-CFTR nasal and bronchial epithelial cell cultures (figure 3a-c). Although $I_{\mathrm{eq}}$ CFTRinh-172 following acute S-VX-445 and FSK addition did not show a significant difference compared with acute FSK addition alone in primary nasal epithelial cells (figure 3d), mainly due to the variable levels of constitutive CFTR conductance, acute S-VX-445 application significantly increased $I_{\text {eq }}$ CFTRinh-172 in primary bronchial epithelial cells (figure 3e). Similarly, acute addition of $S$-VX-445 increased the FSK response of F508del-CFTR after VX-661 rescue in nasal epithelial cultures (figure $3 \mathrm{f}-\mathrm{h}$ ). Pilot studies suggest that the potentiator activity of VX-445 is modified by the phosphorylation status of CFTR. We did not observe potentiation of F508del-CFTR after VX-661 rescue by $S$-VX-445 if it was added after activation with $10 \mu \mathrm{M}$ FSK (data not shown). However, as shown in supplementary figure S2, we did observe a modest but significant potentiation after addition of $0.1 \mu \mathrm{M}$ FSK in WT-CFTR nasal cultures. Interestingly, a similar observation was reported by the McCarty group showing that VX-770 potentiation activity of CFTR variants is influenced by phosphorylation level [34].

We studied the dose dependence of VX-445 potentiation for F508del-CFTR and the rare class II mutants in HEK293 cells after their correction at low-temperature $\left(27^{\circ} \mathrm{C}\right)$ incubation (figure $4 \mathrm{a}$ and b). Of the rare mutants studied, only G85E failed be corrected at low temperature [12]. In this system, VX-445-mediated potentiation of FSK $(10 \mu \mathrm{M})$-activated channel function of F508del-CFTR was measured using the FLIPR membrane potential dye assay as it facilitated the study of multiple doses simultaneously. For F508del-CFTR, the efficacy of VX-445 as a potentiator was significantly less than VX-770, but this was not the case for all of the mutants studied. In figure $4 \mathrm{c}$ and $\mathrm{d}$, we show dose-response curves for VX-770 and VX-445 in potentiating M1101K and N1303K. Interestingly, there were differences in the potentiator responses across the F508del, M1101K and N1303K genotypes. The efficacy of VX-445 as a potentiator was less than that of VX-770 for N1303K but not for $\mathrm{M} 1101 \mathrm{~K}$, where the efficacies for the two compounds were similar. Future studies are required to understand the structural basis for the differential potentiator responses among genotypes.

Next, we studied the potentiation activity of VX-445 on F508del, M1101K, G85E and N1303K mutants expressed in HEK293 cells after pre-treatment with VX-661+S-VX-445 at $37^{\circ} \mathrm{C}$ (figure 5). As shown in figure 5a and b, after correction of F508del-CFTR with VX-661 and $S$-VX-445, the subsequent addition of VX-770 or S-VX-445 potentiated its FSK-dependent channel activity. Regarding the rare mutations, we 

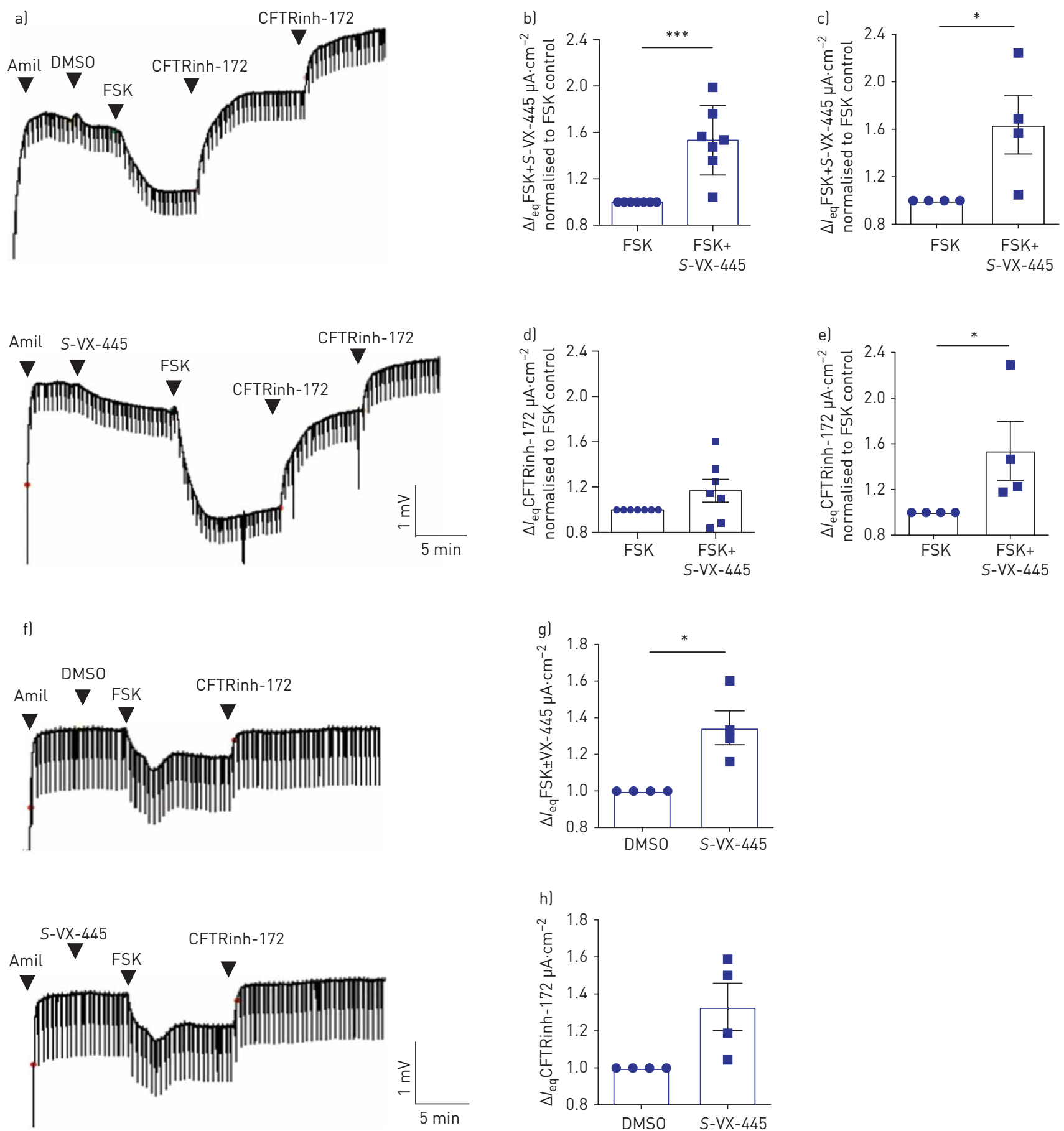

FIGURE 3 VX-445 increased channel activation of wild-type (WT) cystic fibrosis transmembrane conductance regulator (CFTR) in nasal and bronchial epithelial cells. Amil: amiloride; DMSO: dimethyl sulfoxide; FSK: forskolin; leq: transepithelial current. al Representative tracings show Ussing chamber measurements of CFTR function in nasal epithelial cell cultures from a non-CF donor (WT-CFTR). b, c) Fold increase in FSK $(10 \mu \mathrm{M})+S-V X-445(3 \mu \mathrm{M})$-activated $\Delta l_{\text {eq }}$ compared with FSK $(10 \mu \mathrm{M})$ control in b) one to two technical replicates of nasal epithelial cells generated from four healthy controls and c) bronchial epithelial cells from four donors $(n=4)$. d, e) Fold increase in $l_{\text {eq }}$ by the CFTR inhibitor CFTRinh-172 $(10 \mu \mathrm{M})$ in $\mathrm{d})$ nasal epithelial cultures and e) bronchial epithelial cell cultures. f) Representative tracings show Ussing chamber measurements of CFTR function in nasal epithelial cell cultures from a CF patient homozygous for the F508del mutation following treatment with $48 \mathrm{~h}$ of VX-661 $(3 \mu \mathrm{M})$ (F508del-CFTR). CFTRinh-172 $(10 \mu \mathrm{M})$ was given repeatedly to ensure complete inhibition of CFTR. g) Fold increase in FSK (10 $\mu \mathrm{M})+$ S-VX-445 $(3 \mu \mathrm{M})$-activated $\Delta l_{\text {eq }}$ compared with FSK $(10 \mu \mathrm{M})$ control in two technical replicates of nasal epithelial cells generated from two CF donors homozygous for F508del. h) Corresponding fold increase in $l_{\text {eq }}$ by CFTRinh-172 $(10 \mu \mathrm{M})$. Data are presented as mean \pm SD. Comparative analysis was performed using the paired two-tailed t-test $(*: p<0.05 ; * * *: p<0.001)$. Statistically significant differences are shown between untreated cells and those with acute treatment of S-VX-445. 


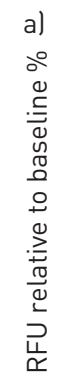
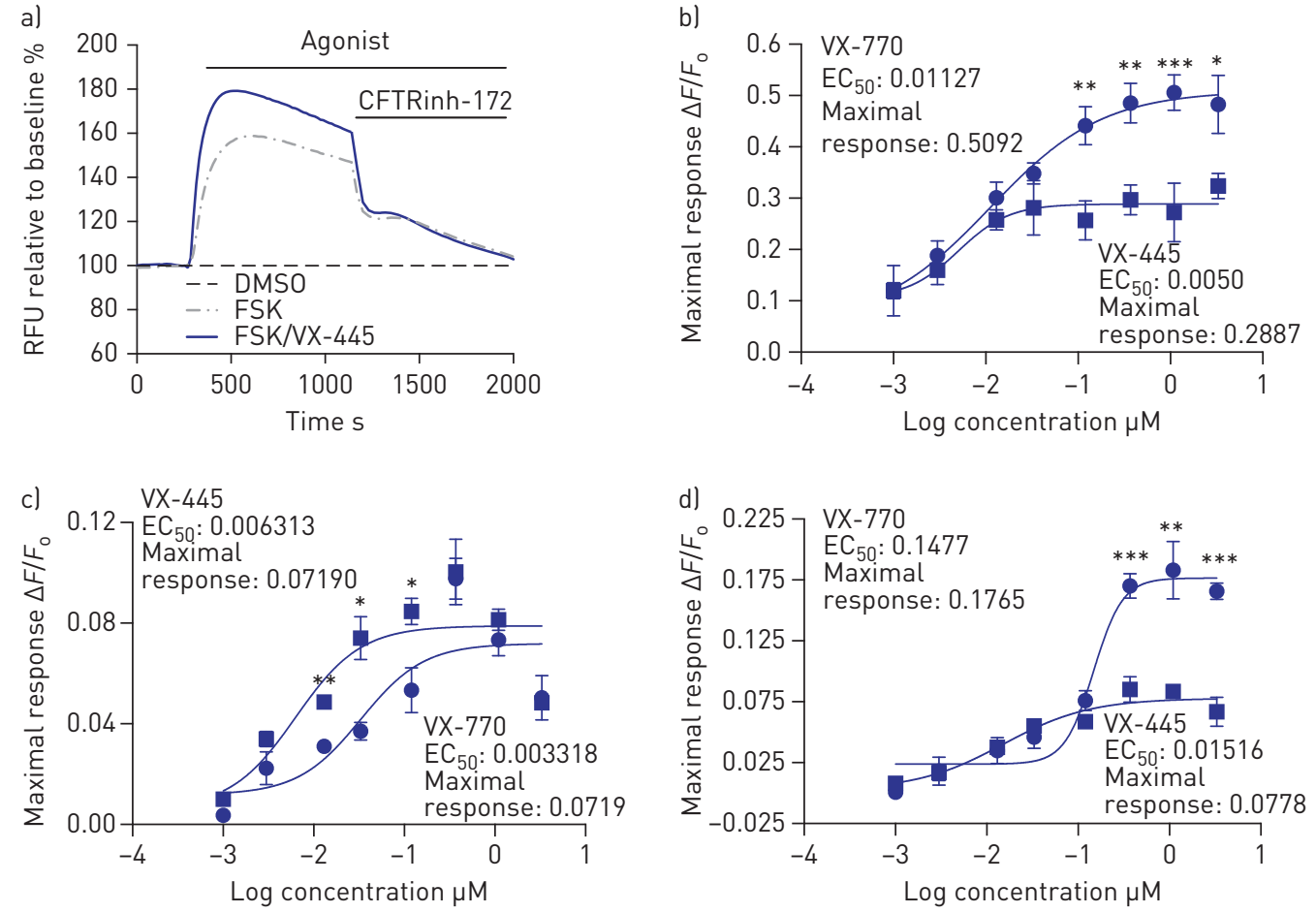

FIGURE 4 VX-445 potentiates temperature-rescued F508del, M1101K and N1303K cystic fibrosis transmembrane conductance regulator (CFTR) mutations in HEK293 cells. RFU: relative fluorescence units; DMSO: dimethyl sulfoxide; FSK: forskolin; $E_{50}$ : half-maximal effective concentration ( $\mu M$ ). a) Representative traces of F508del-CFTR-dependent chloride efflux (membrane depolarisation assay, $3 \mu \mathrm{M} S-\mathrm{VX}-445+10 \mu \mathrm{M}$ FSKJ in HEK293 cells stably transfected with F508del-CFTR after $24 \mathrm{~h}$ incubation at $27^{\circ} \mathrm{C}$. b-d) Doseresponse curves of $V X-770$ or $S-V X-445(0.001-3 \mu M)+F S K(10 \mu M)$ in b) F508del-, c) M1101K- or d) N1303K-CFTR HEK293 cells ( $n=5$ biological replicates and four technical replicates for each experiment). The peak changes in fluorescence to CFTR agonists were normalised relative to the baseline fluorescence $\left(\Delta F / F_{0}\right)$. Data are presented as mean \pm SD. Analysis was performed using one-way ANOVA followed by Turkey's post hoc test (*: $p<0.05 ; * *: p<0.01 ; * * *: p<0.001)$.

confirmed the previous findings in nasal epithelial cell cultures, where VX-809 and VX-661 alone did not rescue VX-770 potentiated channel function, but the combination of VX-445 and VX-661 did rescue VX-770 potentiated activity for M1101K, G85E and N1303K (figure 5c). A similar rescue effect was observed using 16HBE14o- cells edited using CRISPR/Cas9 to express the N1303K-CFTR mutant (CFF 16HBEge N1303K-CFTR) (supplementary figure S3a and b). Interestingly, S-VX-445 also significantly potentiated the FSK-activated function of VX-661+VX-445-corrected rare variants.

Western blot analysis of HEK293 cells expressing CFTR mutants recapitulated our findings on patient-derived nasal cells (figure 5d). Treatment with the corrector VX-809 or VX-661 caused a modest improvement in processing for F508del-CFTR- but not for M1101K- or G85E-CFTR-expressing cells (figure $5 \mathrm{~d}$ and e). Interestingly, and similarly to what we saw in nasal cells, treatment with $S$-VX-445+ VX-661 did not lead to an increase in mature CFTR protein for N1303K. Consistent with our findings in HEK293 cells overexpressing N1303K-CFTR, N1303K-CFTR-expressing 16HBEge cells also showed functional rescue following treatment with $S$-VX-445+VX-661+VX-770 without showing an increase in CFTR protein maturation (supplementary figure S3c and d). We suggest that there is a sufficient amount of mature glycosylated N1303K-CFTR protein to mediate potentiator-induced CFTR channel activity. However, the protein signal of the N1303K-CFTR C band is diffuse in CFF 16HBEge N1303K-CFTR and primary nasal epithelial cells, as reported earlier [35].

Lastly, we tested the effect of S-VX-445+VX-661+VX-770 in nasal cells from one patient homozygous for Y596D/Y596D (supplementary figure S4) and from two patients heterozygous for G542X/N1303K (supplementary figure S5). As shown in supplementary figures S4 and S5, the drug combination S-VX-445+ VX-661+VX-770 did not elicit any increase of CFTR function in either of these mutations.

To summarise, we observed various effects of the $S$-VX-445+VX-661+VX-770 triple combination on the functional rescue of different class II mutations (figure 2i). 

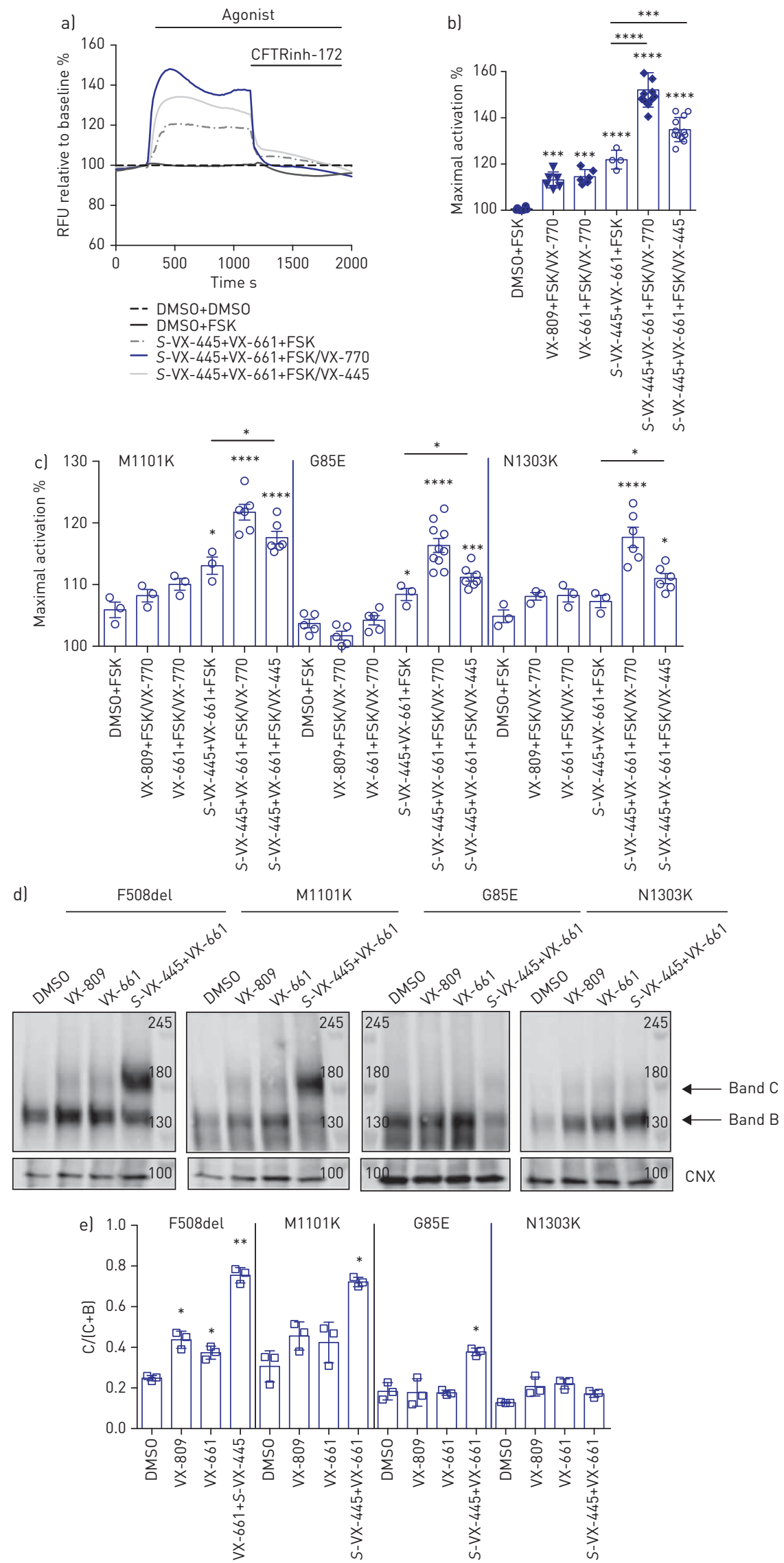
FIGURE 5 F508del cystic fibrosis transmembrane conductance regulator (CFTR) mutation and other rare CFTR mutations expressed in HEK293 cells rescued by VX-445+VX-661+VX-770. RFU: relative fluorescence units; DMSO: dimethyl sulfoxide; FSK: forskolin; CNX: calnexin. al Representative traces of F508del-CFTR-dependent chloride efflux (membrane depolarisation assay) in HEK293 cells stably transfected with F508del-CFTR pre-treated with DMSO or S-VX-445 $(3 \mu \mathrm{M})+V X-661(3 \mu \mathrm{M})$ for $24 \mathrm{~h}$ at $37^{\circ} \mathrm{C}$. b) Maximal activation of F508del-CFTR after stimulation by FSK $(10 \mu \mathrm{M}) \pm V X-770(1 \mu \mathrm{M})$ and/or $S-V X-445(1 \mu \mathrm{M})$. c) HEK293 cells were transiently transfected with M1101K-, G85E- or N1303K-CFTR and treated for $24 \mathrm{~h}$ at $37^{\circ} \mathrm{C}$ with DMSO, VX-809 $(3 \mu \mathrm{M}), \mathrm{VX}-661(3 \mu \mathrm{M})$ or S-VX-445 $(3 \mu \mathrm{M})+\mathrm{VX}-661(3 \mu \mathrm{M})$. Maximal activation of M1101K-, G85E- and N1303K-CFTR after stimulation by FSK $(10 \mu \mathrm{M}) \pm \mathrm{VX}-770(1 \mu \mathrm{M})$ and/or S-VX-445 $(1 \mu \mathrm{M})$ (n=3-10). d) Immunoblots of steady-state expression of F508del-, M1101K-, G85E- or N1303K-CFTR following treatments for $24 \mathrm{~h}$ at $37^{\circ} \mathrm{C}$ with DMSO, VX-809 $(3 \mu \mathrm{M})$, VX-661 $(3 \mu \mathrm{M})$ or S-VX-445 $(3 \mu \mathrm{M})+V X-661(3 \mu \mathrm{M})$. Markers in $\mathrm{kDa}$. Band $\mathrm{C}$ : mature, complex-glycosylated CFTR; band $\mathrm{B}$ : immature, core-glycosylated CFTR. e) Ratio band $C /($ band $C+$ band $B$ ) ( $n=3)$. Data are presented as mean \pm sD. Comparative analysis was performed using one-way ANOVA followed by Turkey's post hoc test $\left(*: p<0.05 ;{ }^{* *}: p<0.01 ;{ }^{* * *}: p<0.001 ;{ }^{* * *}: p<0.0001\right)$.

\section{Discussion}

In this study we show that the novel triple combination of CFTR modulators, i.e. two corrector compounds (VX-445 and VX-661) and the potentiator compound (VX-770), contained in Trikafta improved processing and channel activity of F508del-CFTR in nasal epithelial cell cultures generated from patients homozygous for the major mutation. This result replicates previous pre-clinical data obtained using primary bronchial epithelial cell cultures [7] and supports the use of primary nasal epithelial cell cultures to study modulator efficacy. Interestingly, in the current study we found that the in vitro responses to the triple combination were variable among class II mutation genotypes. These findings prompted in-depth studies of elexacaftor (VX-445) in nasal epithelial cell cultures, wherein we determined that it exhibits two activities, both as a corrector and as a potentiator. Furthermore, these studies support the concept that either or both of elexacaftor's activities could contribute to its rescue effect, depending on the genotype. Given the relative inaccessibility of primary bronchial epithelial cell cultures from individuals who are homozygous for rare processing mutations, our findings support the use of patient-derived nasal epithelial cell cultures for pre-clinical studies of therapeutic interventions as well as studies on their mechanism of action.

Treatment of CF patients homozygous for F508del with the triple combination Trikafta was highly efficacious in clinical trials, leading to an improvement in lung function with an increase of $10 \%$ in percentage predicted forced expiratory volume in $1 \mathrm{~s}$, a decrease in sweat chloride concentration by $45 \mathrm{mmol} \cdot \mathrm{L}^{-1}$ and an improvement in life quality [36]. In pre-clinical studies using primary bronchial epithelial cell cultures, this combination led to a significant functional rescue of the F508del mutant, which exceeded the rescue achieved with Orkambi [7]. Hence, the pre-clinical response size in the bronchial epithelial culture model was predictive of the clinical effect size. Similarly, the in vitro response to this combination in primary nasal epithelial cell cultures from patients homozygous for F508del-CFTR may also provide a biomarker of clinical response. Additional subjects need to be studied, but we propose that the in vitro rescue of F508del/F508del nasal epithelial cell cultures by the triple combination may set the threshold with which to predict clinical efficacy for individuals bearing rare mutations.

Our comparison of VX-770-potentiated FSK-dependent currents and the corresponding CFTRinh-172-sensitive currents in cultures treated chronically with the triple combination (figure 2i) showed that there were variable responses across different genotypes. Interestingly, patient-derived nasal epithelial cell cultures expressing M1101K exhibited a superior functional rescue relative to F508del. Nasal epithelial cell cultures expressing G85E and N1303K exhibited a smaller response. The corresponding studies of mutant protein processing provide insight into possible mechanisms underlying these genotype-specific differences. The triple combination caused a significant increase in protein processing relative to pre-treatment with Orkambi (i.e. VX-809 plus VX-770) for F508del, M1101K and G85E, but not for N1303K. The molecular mechanisms underlying correction by VX-445 were investigated in a recent study by the Lukacs group [37]. VX-445 stabilised NBD1 in biophysical studies; the authors suggested that it acts directly on this domain in the context of the full-length protein. Its efficacy in correcting rare class II mutations residing in distinct domains supports the idea that it has an allosteric effect to promote multidomain assembly. The findings of the current article support these observations and the putative allosteric mechanism of action of VX-445. Future studies are required to determine if differential protein stability at the cell surface accounts for the discrepancy between the magnitude of functional responses to chronic treatment with VX-445+VX-661+VX-770 seen for M1101K and F508del in nasal epithelial cell cultures versus HEK293 cells.

Interestingly, for N1303K-CFTR, there was no improvement in protein processing caused by the triple combination relative to VX-809+VX-770 despite a relative improvement in functional rescue. We suggest that this functional rescue by VX-445+VX-661+VX-770 reflects potentiation of very low residual surface 
expression. This potentiation was mediated by both VX-770 and VX-445 (the dual-acting corrector/ potentiator compound). Interestingly, PHUAN et al. [38] also showed functional rescue of N1303K in an HBE cell line expressing N1303K CFTR with the addition of two potentiators alone, with no corrector [38]. They also showed that N1303K-CFTR was effectively potentiated using a combination of specific potentiation modulators in Fischer thyroid rat cells as well as in nasal and bronchial epithelial cells from a N1303K/N1303K patient [38, 39]. Recently, we also showed that another combination of molecules provided by AbbVie Pharmaceuticals, a potentiator (referred as AP2) plus a dual-acting corrector/ potentiator compound (referred as AC2.2), evoked functional rescue of N1303K-CFTR without a significant improvement in its processing [35]. Altogether, we are seeing a consistent response of N1303K-CFTR to modulators and these findings support the claim that it is the potentiator activity of VX-445 that is important for functional rescue of this mutant.

In summary, we demonstrated variable responses among class II genotypes to elexacaftor, tezacaftor and ivacaftor, while identifying the dual activities of elexacaftor. Our study also highlighted the utility of patient-derived nasal epithelial cell cultures to test drug efficacy and to glean insights regarding the mechanisms underlying drug activity.

Author contributions: O. Laselva, T.J. Moraes, C.E. Bear and T. Gonska designed the experiments. O. Laselva and C. Bartlett performed the experiments and analysed the data. H. Ouyang and T.N.A. Gunawardena cultured patient samples. O. Laselva, C.E. Bear and T. Gonska wrote the paper. T.J. Moraes and P.D.W. Eckford edited the paper.

Conflict of interest: O. Laselva has nothing to disclose. C. Bartlett has nothing to disclose. T.N.A. Gunawardena has nothing to disclose. H. Ouyang has nothing to disclose. P.D.W. Eckford has nothing to disclose. T.J. Moraes has nothing to disclose. C.E. Bear reports grants from CF Canada and Canadian Institutes of Health, during the conduct of the study; a philanthropic donation to the author's host institution from Jagle Bash, outside the submitted work. T. Gonska reports grants from CF Canada/SickKids Foundation, during the conduct of the study; travel reimbursement for lectures and for participation on an advisory board from Vertex Pharmaceutical Inc., outside the submitted work.

Support statement: Primary nasal cell cultures were obtained through the CF Canada-SickKids Program for Individualised CF Therapy (CFIT). This work was supported by the CFIT Program with funding provided by CF Canada and the SickKids Foundation. This work was funded by the Government of Canada through Genome Canada and the Ontario Genomics Institute (OGI-148). This study was funded by the Government of Ontario. Funding information for this article has been deposited with the Crossref Funder Registry.

\section{References}

1 Riordan JR, Rommens JM, Kerem B, et al. Identification of the cystic fibrosis gene: cloning and characterization of complementary DNA. Science 1989; 245: 1066-1073.

2 Bear CE, Li CH, Kartner N, et al. Purification and functional reconstitution of the cystic fibrosis transmembrane conductance regulator (CFTR). Cell 1992; 68: 809-818.

3 Rowe SM, Miller S, Sorscher EJ. Cystic fibrosis. N Engl J Med 2005; 352: 1992-2001.

4 Wainwright CE, Elborn JS, Ramsey BW, et al. Lumacaftor-ivacaftor in patients with cystic fibrosis homozygous for Phe508del CFTR. N Engl J Med 2015; 373: 220-231.

5 Ramsey BW, Davies J, McElvaney NG, et al. A CFTR potentiator in patients with cystic fibrosis and the G551D mutation. N Engl J Med 2011; 365: 1663-1672.

6 Taylor-Cousar JL, Munck A, McKone EF, et al. Tezacaftor-ivacaftor in patients with cystic fibrosis homozygous for Phe508del. N Engl J Med 2017; 377: 2013-2023.

7 Keating D, Marigowda G, Burr L, et al. VX-445-tezacaftor-ivacaftor in patients with cystic fibrosis and one or two Phe508del alleles. N Engl J Med 2018; 379: 1612-1620.

8 Guerra L, Favia M, Di Gioia S, et al. The preclinical discovery and development of the combination of ivacaftor +tezacaftor used to treat cystic fibrosis. Expert Opin Drug Discov 2020; 15: 873-891.

9 Middleton PG, Mall MA, Dřevínek P, et al. Elexacaftor-tezacaftor-ivacaftor for cystic fibrosis with a single Phe508del allele. N Engl J Med 2019; 381: 1809-1819.

10 Molinski S, Eckford PD, Pasyk S, et al. Functional rescue of F508del-CFTR using small molecule correctors. Front Pharmacol 2012; 3: 160

11 Rapino D, Sabirzhanova I, Lopes-Pacheco M, et al. Rescue of NBD2 mutants N1303K and S1235R of CFTR by small-molecule correctors and transcomplementation. PLoS One 2015; 10: e0119796.

12 Lopes-Pacheco M, Boinot C, Sabirzhanova I, et al. Combination of correctors rescues CFTR transmembrane-domain mutants by mitigating their interactions with proteostasis. Cell Physiol Biochem 2017; 41: 2194-2210.

13 Liu Q, Sabirzhanova I, Yanda MK, et al. Rescue of CFTR NBD2 mutants N1303K and S1235R is influenced by the functioning of the autophagosome. J Cyst Fibros 2018; 17: 582-594.

14 DeStefano S, Gees M, Hwang TC. Physiological and pharmacological characterization of the N1303K mutant CFTR. J Cyst Fibros 2018; 17: 573-581.

15 Han ST, Rab A, Pellicore MJ, et al. Residual function of cystic fibrosis mutants predicts response to small molecule CFTR modulators. JCI Insight 2018; 3: e121159.

16 Eckford PDW, McCormack J, Munsie L, et al. The CF Canada-Sick Kids Program in individual CF therapy: a resource for the advancement of personalized medicine in CF. J Cyst Fibros 2019; 18: 35-43.

17 Laselva O, Moraes TJ, He G, et al. The CFTR mutation c.3453G > C (D1152H) confers an anion selectivity defect in primary airway tissue that can be rescued by ivacaftor. J Pers Med 2020; 10: 40. 
18 Laselva O, Erwood S, Du K, et al. Activity of lumacaftor is not conserved in zebrafish Cftr bearing the major cystic fibrosis-causing mutation. FASEB Bioadv 2019; 1: 661-670.

19 Valley HC, Bukis KM, Bell A, et al. Isogenic cell models of cystic fibrosis-causing variants in natively expressing pulmonary epithelial cells. J Cyst Fibros 2019; 18: 476-483.

20 Wu YS, Jiang J, Ahmadi S, et al. Orkambi-mediated rescue of mucociliary clearance in cystic fibrosis primary respiratory cultures is enhanced by arginine uptake, arginase inhibition, and promotion of nitric oxide signaling to the cystic fibrosis transmembrane conductance regulator channel. Mol Pharmacol 2019; 96: 515-525.

21 Cao $\mathrm{H}$, Ouyang $\mathrm{H}$, Laselva $\mathrm{O}$, et al. A helper-dependent adenoviral vector rescues CFTR to wild type functional levels in CF epithelial cells harbouring class I mutations. Eur Respir J 2020; 56: 2000205.

22 Molinski SV, Ahmadi S, Ip W, et al. Orkambi and amplifier co-therapy improves function from a rare CFTR mutation in gene-edited cells and patient tissue. EMBO Mol Med 2017; 9: 1224-1243.

23 Laselva O, McCormack J, Bartlett C, et al. Preclinical studies of a rare CF-causing mutation in the second nucleotide binding domain $($ c.3700A $>\mathrm{G})$ show robust functional rescue in primary nasal cultures by novel CFTR modulators. J Pers Med 2020; 10: E209.

24 Erwood S, Laselva O, Bily TMI, et al. Allele-specific prevention of nonsense-mediated decay in cystic fibrosis using homology-independent genome editing. Mol Ther Methods Clin Dev 2020; 17: 1118-1128.

25 Laselva O, Stone TA, Bear CE, et al. Anti-infectives restore ORKAMBI rescue of F508del-CFTR function in human bronchial epithelial cells infected with clinical strains of $P$. aeruginosa. Biomolecules 2020; 10: 334 .

26 Cao H, Ouyang H, Ip W, et al. Testing gene therapy vectors in human primary nasal epithelial cultures. Mol Ther Methods Clin Dev 2015; 2: 15034.

27 Laselva O, Eckford PD, Bartlett C, et al. Functional rescue of c.3846G >A (W1282X) in patient-derived nasal cultures achieved by inhibition of nonsense mediated decay and protein modulators with complementary mechanisms of action. J Cyst Fibros 2020; 19: 717-727.

28 Chin S, Ramjeesingh M, Hung M, et al. Cholesterol interaction directly enhances intrinsic activity of the cystic fibrosis transmembrane conductance regulator (CFTR). Cells 2019; 8: 804 .

29 Laselva O, Marzaro G, Vaccarin C, et al. Molecular mechanism of action of trimethylangelicin derivatives as CFTR modulators. Front Pharmacol 2018; 9: 719.

30 Molinski SV, Shahani VM, Subramanian AS, et al. Comprehensive mapping of cystic fibrosis mutations to CFTR protein identifies mutation clusters and molecular docking predicts corrector binding site. Proteins 2018; 86: 833-843.

31 Laselva O, Molinski S, Casavola V, et al. Correctors of the major cystic fibrosis mutant interact through membrane-spanning domains. Mol Pharmacol 2018; 93: 612-618.

32 Cholon DM, Quinney NL, Fulcher ML, et al. Potentiator ivacaftor abrogates pharmacological correction of DeltaF508 CFTR in cystic fibrosis. Sci Transl Med 2014; 6: 246 ra96.

33 Veit G, Avramescu RG, Perdomo D, et al. Some gating potentiators, including VX-770, diminish DeltaF508-CFTR functional expression. Sci Transl Med 2014; 6: 246 ra97.

34 Cui G, Stauffer BB, Imhoff BR, et al. VX-770-mediated potentiation of numerous human CFTR disease mutants is influenced by phosphorylation level. Sci Rep 2019; 9: 13460.

35 Laselva O, Bartlett C, Popa A, et al. Emerging preclinical modulators developed for F508del-CFTR have the potential to be effective for ORKAMBI resistant processing mutants. J Cyst Fibros 2021; 20: 106-119.

36 Heijerman HGM, McKone EF, Downey DG, et al. Efficacy and safety of the elexacaftor plus tezacaftor plus ivacaftor combination regimen in people with cystic fibrosis homozygous for the F508del mutation: a double-blind, randomised, phase 3 trial. Lancet 2019; 394: 1940-1948.

37 Veit G, Roldan A, Hancock MA, et al. Allosteric folding correction of F508del and rare CFTR mutants by elexacaftor-tezacaftor-ivacaftor (Trikafta) combination. JCI Insight 2020; 5: e139983.

38 Phuan PW, Tan JA, Rivera AA, et al. Nanomolar-potency 'co-potentiator' therapy for cystic fibrosis caused by a defined subset of minimal function CFTR mutants. Sci Rep 2019; 9: 17640.

39 Phuan PW, Son JH, Tan JA, et al. Combination potentiator ('co-potentiator') therapy for CF caused by CFTR mutants, including N1303K, that are poorly responsive to single potentiators. J Cyst Fibros 2018; 17: 595-606. 